\title{
Critical role of extracellularly secreted neuronal pentraxin 1 in ischemic neuronal death
}

\author{
Shabarish Thatipamula ${ }^{1}$ and Mir Ahamed Hossain ${ }^{1,2,3^{*}}$
}

\begin{abstract}
Background: Developing brain is highly susceptible to hypoxic-ischemic injury leading to severe neurological disabilities in surviving infants and children. Previously we reported induction of neuronal pentraxin 1 (NP1) in hypoxic-ischemic injury in neonatal brain and NP1 co-localization with the excitatory AMPA receptors GluR1 at the synaptic sites. However, how NP1 contributes to hypoxic-ischemic neuronal injury is not completely understood.

Results: Here we report that extracellular secretion of NP1 is required for ischemic neuronal death. Primary cortical neurons at days in vitro (DIV) 12 were subjected to oxygen glucose deprivation (OGD), an in vitro model of ischemic stroke, for different time periods (2 8 h). Oxygen glucose deprivation showed characteristic morphological changes of dying cells, OGD time-dependent induction of NP1 (2-4-fold) and increased neuronal death. In contrast, the NP1-KO cortical neurons were healthy and showed no sign of dying cells under similar conditions. NP1gene silencing by NP1-specific small interfering RNA (NP1-siRNA) protected cortical neurons from OGD-induced death. Conditioned media (CM) collected from OGD exposed WT cortical cultures caused neurotoxicity when added to a subset of DIV 12 normoxia control WT cortical cultures. In contrast, CM from OGD-exposed NP1-KO cultures did not induce cell toxicity in control WT cultures, suggesting a role for extracellular NP1 in neuronal death. However, NP1-KO neurons, which showed normal neuronal morphology and protection against OGD, sustained enhanced death following incubation with CM from WT OGD-exposed cultures. Western blot analysis of OGD exposed WT CM showed temporal increase of NP1 protein levels in the CM. Most strikingly, in contrast to NP1-KO CM, incubation of normal cortical cultures with CM from OGD exposed NP2-KO cultures showed neurotoxicity similar to that observed with CM from OGD exposed WT neuronal cultures. Western immunoblotting further confirmed the increased presence of NP1 protein in OGD-exposed NP2-KO CM. Live immunofluorescence analysis show intense cell surface clustering of NP1 with AMPA GluR1 receptors.
\end{abstract}

Conclusions: Collectively, our results demonstrate that extracellular release of NP1 promote hypoxic-ischemic neuronal death possibly via surface clustering with GluR1 at synaptic sites and that NP1, not its family member NP2, is involved in the neuronal death mechanisms.

Keywords: Oxygen glucose deprivation, Neuronal pentraxin 1, Conditioned medium, Neuronal death, Primary cortical neurons, Synapse, AMPA receptor GluR1

\section{Background}

Neuronal injury occurring with cerebral hypoxia-ischemia (HI) has been attributed to overstimulation of $\mathrm{N}$-methylD-aspartate (NMDA) and $\alpha$-amino-3-hydroxy-5-methyl4-isoxazole-propionic acid (AMPA) subtypes of glutamate receptors [1-4], oxidative stress, and activation of intrinsic

\footnotetext{
* Correspondence: hossain@kennedykrieger.org

'Hugo W. Moser Research Institute at Kennedy Krieger, Baltimore, MD 21205, USA

2Department of Neurology, Johns Hopkins University School of Medicine, Baltimore, MD 21205, USA

Full list of author information is available at the end of the article
}

program of apoptotic cell death [5]. One of the proteins that is induced under hypoxic-ischemic stress and initiates neuronal death program is neuronal pentraxin $1^{\prime}$ (NP1) [6-8]. However, how NP1 contributes to neuronal death is not completely understood. NP1 is predominantly expressed in the central nervous system [9-12] and belongs to the long pentraxins family of proteins; NP1, neuronal activity-regulated pentraxin (Narp; also called NP2), and neuronal pentraxin receptor (NPR) $[9,12,13]$. Neuronal pentraxins have high homology among human, mouse and rats [14]. The pentraxins have several structural and functional characteristics to form side-

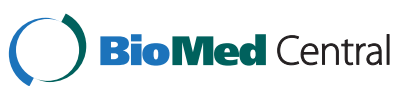

2014 Thatipamula and Hossain; licensee BioMed Central. This is an Open Access article distributed under the terms of the Creative Commons Attribution License (http://creativecommons.org/licenses/by/4.0), which permits unrestricted use, distribution, and reproduction in any medium, provided the original work is properly credited. The Creative Commons Public Domain Dedication waiver (http://creativecommons.org/publicdomain/zero/1.0/) applies to the data made available in this article, unless otherwise stated. 
to-side and head-to-head multimeric aggregates $[13,15,16]$ and the ability to bind other proteins via a lectin-like domain.

Proposed functions of NPs include modulating synaptic uptake, synapse formation, and synaptic remodeling $[9,17]$. NP2 has been reported to mediate synaptic clustering of AMPA glutamate receptors $[18,19]$. In our previous studies, we have shown induction of NP1 in neonatal mice brain following $\mathrm{HI}$ and injury to the cerebral cortex and hippocampal CA 3 and CA1 brain regions $[7,20,21]$. We found that the increase in NP1 induction occurs before the actual cell death, consistent with a role for NP1 in the injury mechanisms. We also found that NP1 co-localizes with AMAP GluR1 receptors and enhanced GluR1 membrane insertion at the synaptic sites as evident by NP1-GluR1-PSD-95 co-clustering following OGD exposure [22]. It is known that various cell death mechanisms require de novo synthesis of both RNA and lethal proteins $[5,23]$, and low neuronal activity triggers the intrinsic program of apoptotic cell death in mature neurons [5]. However, how induction of NP1 expression leads to the propagation of neuronal death or survival of neurons in the absence of NP1 expression is not completely understood. Here, we report that the extracellular secretion of NP1 is required to induce neuronal death in primary cortical neurons subjected to oxygen glucose deprivation (OGD) possibly through co-clustering with APMA GluR1 receptors at synaptic sites and enhanced excitotoxicity. Our findings suggest that blockade of NP1 induction and its extracellular release may be therapeutically relevant against hypoxic-ischemic injury in neonatal brain.

\section{Methods}

Embryonic cortical neuronal culture

The Johns Hopkins University Institutional Animal Care and Use Committee approved all animal protocols used; they complied with the US NIH Guide for the Care and Use of Laboratory Animals. Primary cortical neuronal cultures were prepared from embryonic day 16 (E16) wildtype (WT) and NP1-knockout (NP1-KO) mice as described previously [7]. NP1 knockout mice were provided by Dr. Paul Worley, Dept. of Neuroscience, School of medicine, Johns Hopkins University, Baltimore, MD, USA. Primary cortical neurons were grown in a culture medium consisting of Neurobasal medium (Invitrogen, Carlsbad, CA, USA), 2\% B27 supplement (Invitrogen), 2-mM L-glutamine, and $1 \%$ penicillin-streptomycin as described previously [7]. At 3 days in vitro (DIV), one-third of the media was replaced with fresh medium (without L-glutamine) containing cytosine arabinofuranoside (AraC, $5 \mu \mathrm{M}$; Sigma, St. Louis, MO, USA) to arrest the growth of non-neuronal cells. Experiments were conducted at DIV 12, when cultures consisted primarily of neurons ( $>95 \%$ MAP-2 immunoreactive cells) (MAP-2; Chemicon, Temecula, CA, USA).

\section{Induction of OGD, modeled in vitro, using cultured primary cortical neurons}

To induce oxygen glucose deprived conditions, cultured cortical neurons at DIV 12 were exposed to OGD as described previously $[6,22,24]$. Briefly, neurons were placed in glucose-free Earls balanced salt solution (EBSS) and then exposed to humidified $95 \% \mathrm{~N}_{2} / 5 \% \mathrm{CO}_{2}$ using anaerobic modular incubator chambers (Billups-Rothenberg, Del Mar, CA, USA) for different time periods (2 $8 \mathrm{~h}$ ). Control cultures were incubated with EBSS with glucose and incubated in humidified $95 \%$ air $/ 5 \% \mathrm{CO}_{2}$ for the same duration. After indicated periods of OGD, cells were washed with ice-cold PBS and harvested to examine various biochemical and morphological end points.

\section{SDS-PAGE and western blot analyses}

SDS-PAGE and immunoblotting were performed according to the method as described previously [7,25,26]. Total proteins $\left(\begin{array}{lll}20 & 30 \mu \mathrm{g}\end{array}\right)$ were diluted in Laemmli buffer containing 2-mercaptoethanol, heated to $95 \mathrm{C}$ for $5 \mathrm{~min}$, separated on a 4-20\% gradient Tris-glycine precast gel (Invitrogen) at $120 \mathrm{~V}$ for $1.5 \mathrm{~h}$. Blots were incubated with primary antibodies specific for NP1 (1:500, BD Transduction Laboratories, Tamecula, CA, USA). HRP (horseradish peroxidase)-conjugated secondary antibodies (GE Healthcare, Piscataway, NJ, USA) were used at 1:10000 dilutions for $1 \mathrm{~h}$ at room temperature. The HRP reaction product was visualized using an ECL Western blotting detection kit (GE healthcare). Image films were scanned in gray scale (HP Scanjet G4010) at a high resolution as TIFF files. Immunoreactive protein bands corresponding to the correct molecular mass of target protein were quantified by drawing rectangle around the individual band and the intensity was measured by densitometry using NIH ImageJ software. Values were normalized to internal standard actin, which also serve as a loading control, to make relative comparisons.

\section{Assessment of cell viability/toxicity}

Immediately after the indicated periods of OGD exposure, cell viability and cell death was determined by independent and complementary methods as described previously $[6,7,22,24]$.

\section{MTT assay}

Mitochondrial dehydrogenase activity cleaves 3-(4,5-dimethylthiazol-2-yl)-2,5-diphenyl tetrazolium bromide (MTT; Sigma), which is considered as a biochemical index for cellular viability. A quantitative colorimetric assay of MTT [27] used to determine cell survival as described previously $[7,25]$. The results were expressed as a percentage of control cultures viability. 


\section{LDH assay}

Lactate dehydrogenase (LDH) activity released in the media after OGD exposure was measured using the CytoTox96 Non-radioactive Cytotoxicity Assay kit (Promega, Madison, WI, USA) as described previously $[7,28]$. Percent cell death was determined using the formula: \% cytotoxicity = OGDinduced LDH release $\left(\mathrm{OD}_{490}\right) /$ maximum $\mathrm{LDH}$ release $\left(\mathrm{OD}_{490}\right)$ after correcting for baseline absorbance of $\mathrm{LDH}$ release at $490 \mathrm{~nm}$.

\section{TUNEL staining}

The DeadEnd Fluorometric TUNEL System (Promega) was used to detect cell death in cultured primary cortical neurons exposed to OGD (2-8 h) as described previously $[29,30]$. This method allows direct detection of nuclear DNA fragmentation, an important biochemical hallmark of cell death, by catalytically incorporating fluorescein-12dUTP at $3^{\prime}-\mathrm{OH}$ DNA ends. Primary cortical cultures grown on cover slips were processed according to manufacturers instructions. Negative controls were performed under identical conditions except for the omission of terminal deoxynucleotidyl transferase (TdT) from the reaction buffer. Fluorescein fluorescence was visualized in a fluorescence microscope (Carl Zeiss Axioplan 1) with an excitation at $485 \mathrm{~nm}$ and an emission at $535 \mathrm{~nm}$. DAPI fluorescence (blue) was visualized with an excitation and emission filters at $365 \mathrm{~nm}$ and $450 \mathrm{~nm}$, respectively.

\section{Short interference RNA (siRNA) directed against NP1 mRNA}

For NP1 gene silencing experiments, we have used Ntpx1 specific siRNA constructs (5'-AATTCTTCCAGCCAAA CCAAC-3') (construct \#3) (5-AAGAACGACACAGAGG AAAGG-3') (construct \#5) generated using Silencer siRNA construction kit (Cat \#1620) (Ambion, Inc. Austin, TX, USA) and the commercially available control scramble siRNA (SsiRNA) following methods described previously [8]. The oligodeoxyribonucleotide sequences exhibited no similarity to any other known mammalian genes as determined by BLAST. Experimental treatments were initiated $\sim 48 \mathrm{~h}$ after transfection. Using siRNA specific for NP1, we have achieved $>90 \%$ reduction in NP1 protein levels compared to control SsiRNA.

\section{Quantification of NP1 expression by real-time PCR}

Total RNA was extracted from control and OGDexposed primary cortical cultures using TRIzol reagent (Invitrogen) according to manufacturer s protocol. The cDNA was synthesized from $1 \mu \mathrm{g}$ of purified total RNA using iScript cDNA Synthesis Kit (Bio-Rad laboratories, Richmond, CA, USA), following the manufacturers instructions. Quantitative real-time PCR was performed in triplicate by using iQ SYBR Green Supermix on CFX96 Real-Time System (Bio-Rad) as described previously [6].
The mRNA level was normalized by housekeeping gene HPRT [31]. The primers set used for NP1 (Acc. No. NM_008730.2) were 5'-GCT GCG AGA GCC AGA GCA CC-3' (sense) and 5' ${ }^{\prime}$ TTG CCC GAG TTG GCT GAG CG-3' (anti-sense), and for HPRT were 5'-CCT GGC GTC GTG ATT AGT GAT G-3' (sense) and 5'-C AG AGG GCT ACA ATG TGA TGG C-3' (anti-sense).

\section{Immunofluorescence}

Live double-immunofluorescence staining of primary cortical cultures (DIV 12) with NP1 was done as described previously $[7,22]$. Briefly, cortical neurons, grown on coverslips, following exposure to OGD $(4 \mathrm{~h})$ were live labeled with both anti-NP1 (1:100; BD Transduction Laboratories) and anti-GluR1 (1:100; Millipore) by adding directly to the medium and further incubated for $45 \mathrm{~min}$ at $37 \mathrm{C}$. Neurons were then fixed with $3.7 \%$ formaldehyde, and permeabilized cells were stained with antimouse Alexa Fluor 568 (red, NP1) and anti-rabbit Alexa fluor 488 (green, GluR1)-conjugated secondary antibodies (Invitrogen). Slides were coverslipped with prolong mounting medium containing DAPI (blue) (Molecular Probes, Eugene, OR, USA) to stain nuclei. Immunofluorescence was visualized using an inverted fluorescence microscope (Olympus IX51fitted with DP2-DSW-V3.2 application software) at 10 and ZEISS Axioimager M2 (AxioVision SE64 Rel.4.8.1 application software) at 100 magnification.

\section{Statistical analysis}

Statistics were performed using GraphPad Prism software, Version 5.01. For one experimental and one control group, two-tailed Students $t$-test was used to determine if differences exist between means. Comparisons involving multiple groups were done by ANOVA, followed by Bonferroni/ Dunn post-hoc test where appropriate. Significance level was assigned at $\mathrm{P}<0.05$.

\section{Results}

Induction of NP1 in primary cortical neurons exposed to oxygen glucose deprivation

Primary cortical neuronal cultures at DIV12 were exposed to OGD for indicated times $(28 \mathrm{~h})$. Control cultures were incubated with EBSS containing glucose and exposed to humidified $95 \%$ air $/ 5 \% \mathrm{CO}_{2}$ for the same duration of time. Light microscopic analysis showed that control neurons were healthy and retained normal morphology, as indicated by larger size, phase brightness and intact processes. Whereas, OGD exposed neurons showed characteristic morphological changes of dying cells, which were round, smaller and translucent with disintegration of processes and cell bodies compared to control normoxic neurons (Figure 1). In contrast, NP1-KO cortical cultures retained normal neuronal morphology; intact processes and healthy 


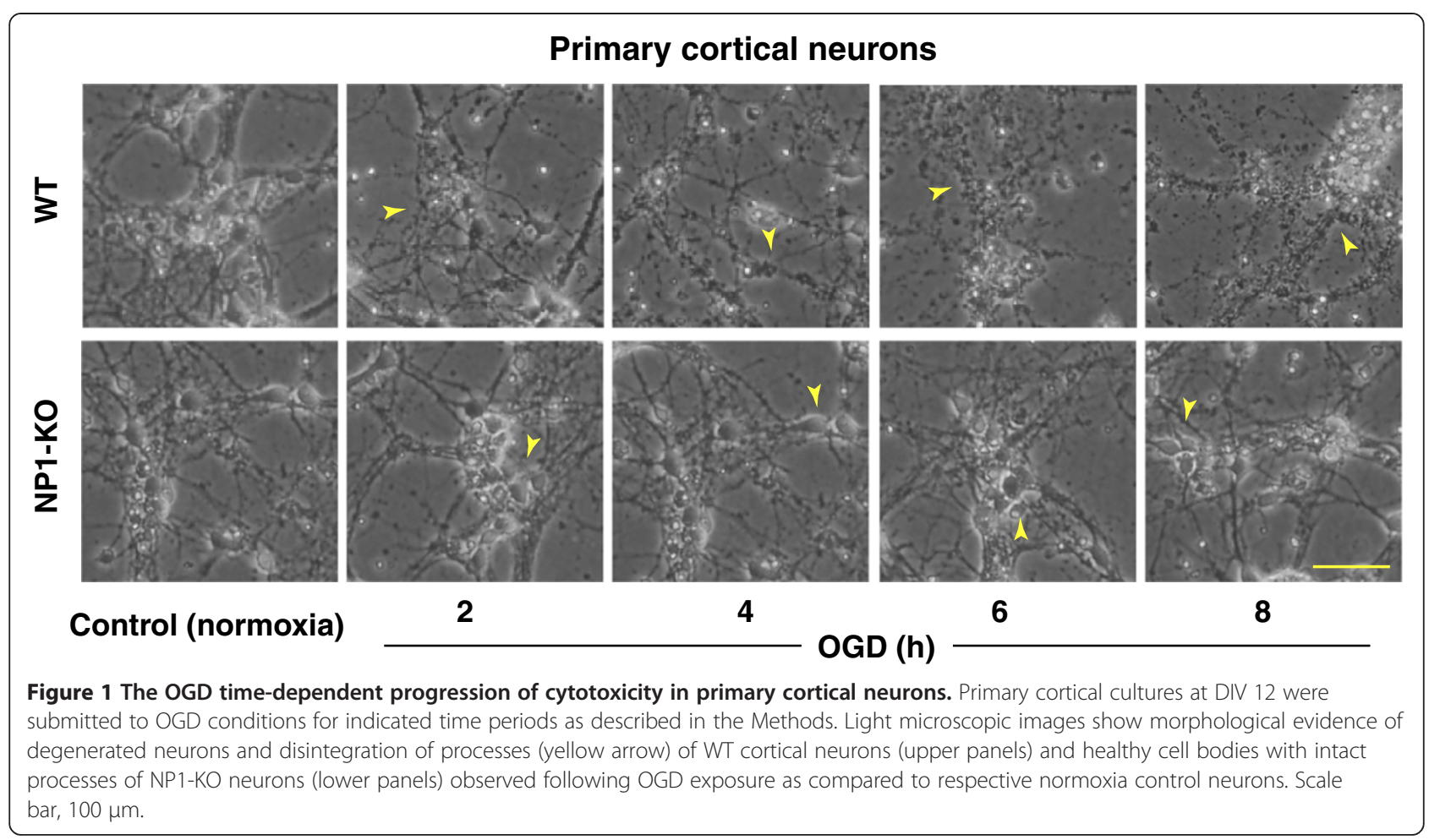

cell bodies against OGD (2 $8 \mathrm{~h}$ ), similar to normoxia control cultures (Figure 1). This in vitro OGD model was used in subsequent experiments to determine the role of NP1 and its specific requirement for ischemic neuronal death.

Next, we asked if NP1 is induced in cultured primary cortical neurons in response to OGD. The RT-qPCR data showed OGD time-dependent increased expressions of NP1 mRNA $(\sim 4$-fold; $\mathrm{p}<0.01)$ compared to that in normoxia controls (Figure 2A). The increased expression of NP1 mRNA was further validated by Western blot analyses (Figure 2B). Western blot analysis of total cellular extracts revealed a NP1-specific immunoreactive protein band with apparent molecular mass of $\sim 47 \mathrm{kDa}$ consistent with the expected size of NP1 $[9,17]$. Quantitative densitometry values of NP1 protein normalized to $\beta$-actin (NP1/ $\beta$-actin ratio, $\mathrm{n}=6)$ further confirmed OGD time-dependent increase of NP1 protein levels, which reached the maximum (4-fold) at $8 \mathrm{~h}$ of OGD, examined. The NP1 protein levels in normoxia controls were low but detectable range.

OGD exposure caused cytotoxicity in WT neurons, whereas, NP1-KO cortical neurons are protected against OGD

Primary cortical neuronal cultures at DIV 12 were exposed to OGD for different time periods $(4,6$, and $8 \mathrm{~h})$. LDH release cytotoxicity assay revealed OGD time-dependent increase in cell death in WT cortical cultures. OGD exposure (4 6 h) resulted $30-50 \%(\mathrm{p}<0.001)$ cell death in WT cultures (Figure $2 \mathrm{C}$ ). In contrast, we observed very negligible change (not significant) in the release of LDH in NP1-KO neurons as compared to the WT cells following OGD exposure. Interestingly, NP1-KO cortical neurons maintained morphological integrity for, at least, $8 \mathrm{~h}$ of OGD exposure, which were consistent with cell cytotoxicity as determined by $\%$ LDH release (Figure $2 \mathrm{D}$ ).

Specific involvement of NP1 in OGD-induced neuronal death To determine the specificity of NP1 induction in neuronal death, we transfected WT primary cortical neurons with either control scramble (SsiRNA) or NP1-siRNA to knockdown NP1 in WT neurons (Figure 3). Using our established protocol, we found that NP1-siRNA almost completely knockdown NP1 protein levels (>90\%) compared to that in cells transfected with control SsiRNA (shown in inset). Here, we asked that if the NP1 induction is directly associated with neuronal death then knocking down of NP1 protein will protect cortical neurons against OGD-induced death. LDH cytotoxicity (Figure 3A), MTT cell viability (Figure $3 \mathrm{~B}$ ) assays and TUNEL (+) staining for degenerated neurons (Figure $3 C$ ) revealed that OGD exposure $(6 \mathrm{~h})$ resulted significant cell death $(\mathrm{p}<0.01)$ in WT neurons transfected with control scramble siRNA. In contrast, primary cortical neurons transfected with NP1siRNA, showed significantly decreased neurotoxicity and degenerated neurons (i.e. neuroprotection) when submitted to OGD exposure ( $6 \mathrm{~h}$ ). Our results clearly demonstrate that NP1 is specifically involved in hypoxic-ischemic neuronal death. 


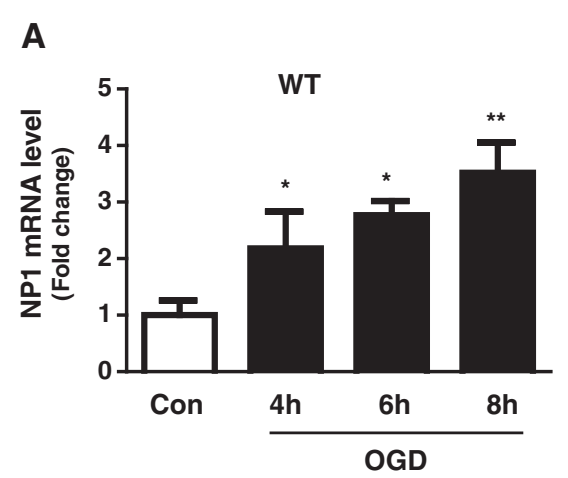

B
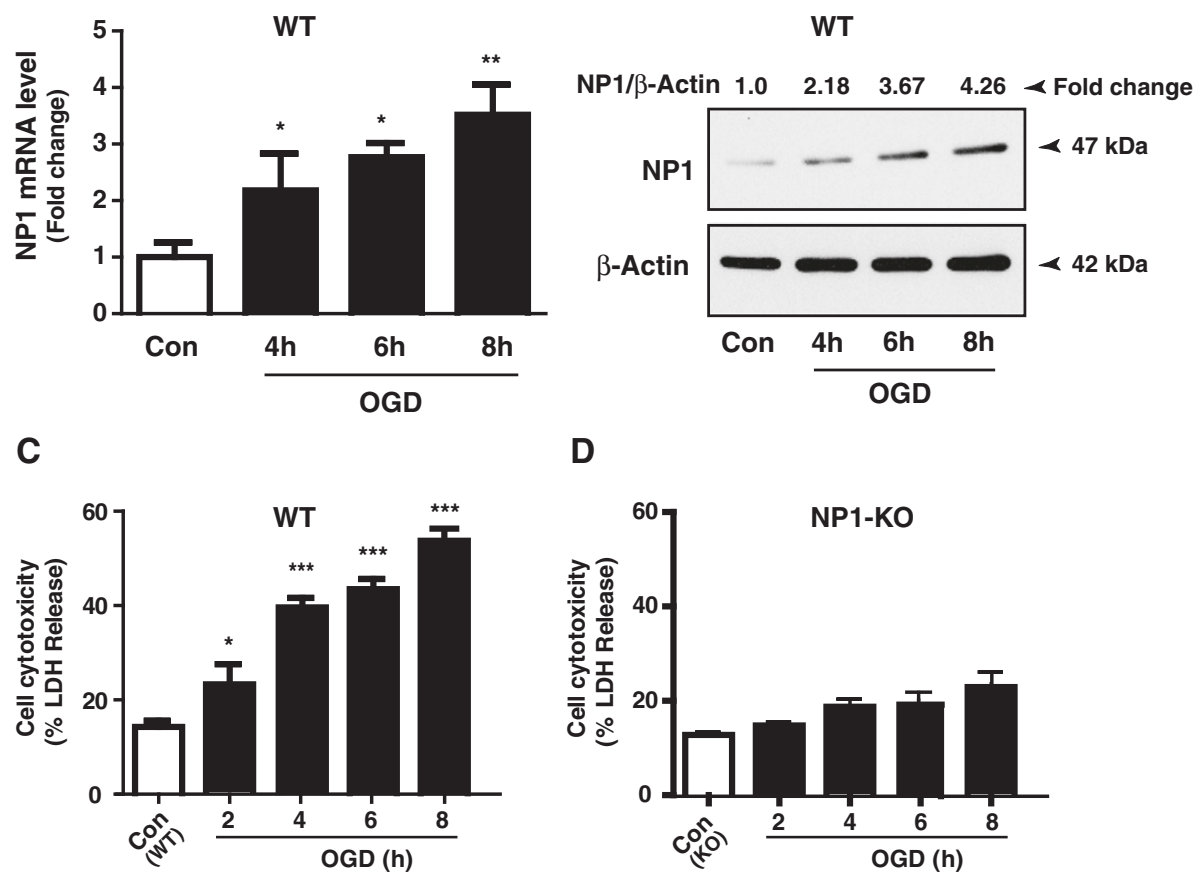

D

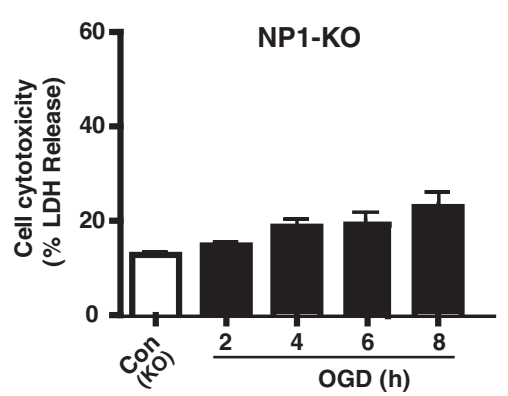

Figure 2 The NP1 induction is associated with OGD-induced cortical neuronal death. A) Total cellular RNA was extracted and NP1 mRNA expression levels were analyzed by RT-qPCR. Data show relative quantification of Nptx 1 expression at different time periods of OGD exposure. Fold induction is the ratio of NP1 to internal control HPRT, which remained stable throughout the OGD period (mean $S E M, n=6$; ${ }^{*} p<0.05$, $\left.{ }^{* *} \mathrm{p}<0.01\right)$. B) Total cellular protein was analyzed by SDS-PAGE and immunoblotted for NP1 protein using NP1-specific antibody that detected NP1-immunoreactive single band of molecular mass $47 \mathrm{kDa}$. The $\beta$-actin serves as loading control. Quantitative densitometry values normalized to $\beta$-actin (NP1/ $\beta$-actin ratio, $n=6$ ) are also shown. Representative bands are shown. $\mathbf{C} \& \mathbf{D}$ ) OGD exposure of WT cortical neurons caused cell death, while NP1-KO neurons were protected against OGD. Quantification of cell death as indicated by LDH release showed OGD time dependent increase of LDH release at 2, 4, 6, and $8 \mathrm{~h}$ of OGD exposures of WT cortical neurons. LDH release remained at the control level or non-significant increase in OGD-exposed NP1-KO neurons. Data are expressed as \% LDH release normalized to normoxia control (mean SEM, $n=8 ;{ }^{*} p<0.05$, *** $p<0.001$ ). We found $\sim 50 \%$ cytotoxicity occurred at $6 \mathrm{~h}$ of $\mathrm{OGD}$.

Enhanced neuronal death induced by conditioned media (CM) from OGD-exposed WT primary cortical cultures Because of the secretory nature of NP1 [9,11-13], we asked if NP1 induction is associated with hypoxicischemic neuronal death, then extracellularly secreted NP1 might be also contributing to the neuronal death observed. We collected the conditioned media (CM) from control and OGD exposed WT cortical cultures (WTOGD CM) and concentrated using Microsep 30K Omega Centrifugal Devices (Cat no. OD030C46; Pall Life Sciences, Ann Arbor, MI, USA). Western blot analysis of WT control and OGD-exposed CM confirmed OGD timedependent increase of NP1 protein levels present in the WT-OGD CM (Figure 4A). After confirming the presence of NP1 in the WT-OGD CM, we added this CM to a subset of control cortical cultures at DIV 12 and incubated for additional $24 \mathrm{~h}$. An additional group of the same CM-treated cultures were also exposed to OGD (6 h). LDH release cytotoxicity assay revealed that addition of WT-OGD CM to control normoxia cultures resulted significant cell death $(\mathrm{P}<0.01)$ similar to that observed with OGD exposed WT cultures (Figure 4B). In addition, OGD exposure of the WT-OGD CM treated neurons further enhanced the cytotoxicity $(\mathrm{p}<0.01)$ (Figure 4B).

To specifically examine the role of NP1 in neuronal death, we used CM collected separately from control, OGDexposed WT (WT-OGD CM) and NP1-KO cortical cultures (NP1-KO OGD CM), concentrated as above, added to separate subsets of WT and NP1-KO cultures at DIV 12, and incubated for additional for $24 \mathrm{~h}$ (Figure 5). Most interestingly, we found that WT-OGD CM caused neuronal death (degeneration of cell bodies and processes as shown by yellow arrows) when added to normal WT cultures (Figure 5 A1). In contrast, the CM from OGD exposed NP1-KO (NP1-KO OGD CM) did not cause any degeneration of processes and cell bodies and maintained normal neuronal morphology (shown by green arrows) when added to the normoxia WT cultures (Figure 5B1) similar to control cultures. On the other hand, WT-OGD CM caused neuronal 
A

C
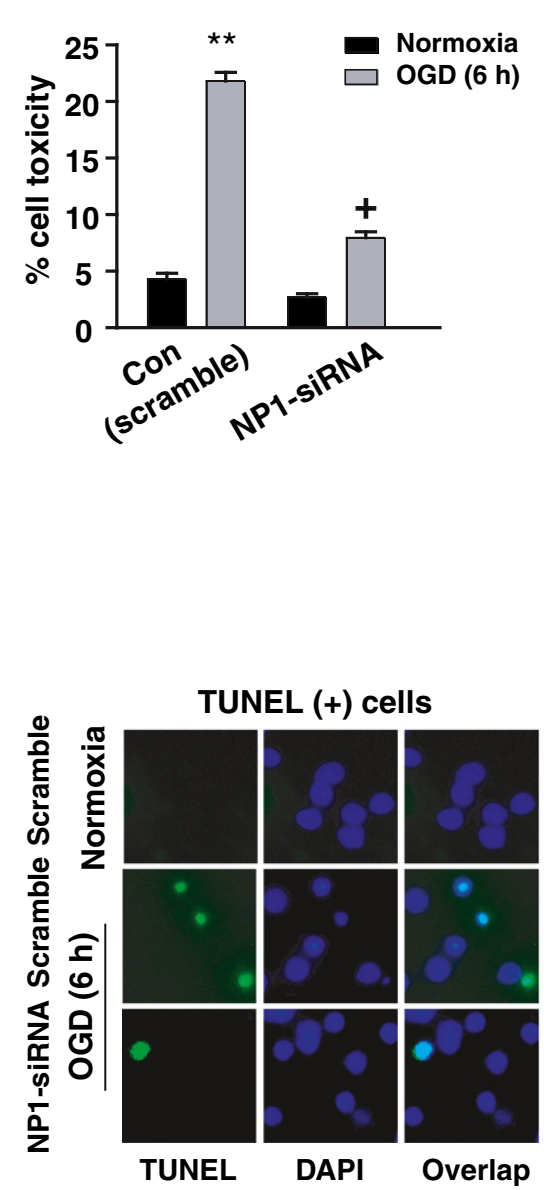

B
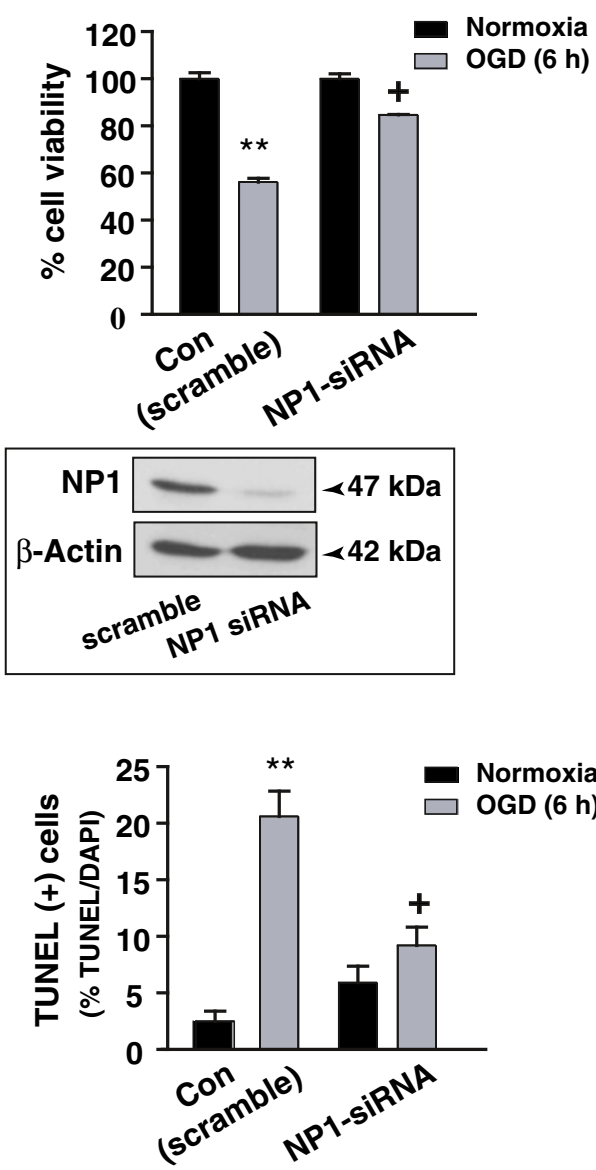

Figure 3 Knockdown of NP1 by siRNA targeted against NP1 mRNA significantly protected against OGD-induced neuronal death. We have achieved $>90 \%$ reduction in NP1 protein compared to scramble siRNA (shown in inset). LDH cytotoxicity and MTT cell viability (A-B) showed significantly less cell death in NP1-siRNA transfected WT cells vs. non-transfected cells following exposure to OGD (6 h). Data are expressed as percent LDH release normalized to normoxia control (mean SEM, $n=8 ;{ }^{* *} p<0.01$ compared to control normoxia; $+p<0.01$ compared to OGD scramble siRNA group) C) TUNEL staining of cortical neurons transfected with either control scramble or NP1-siRNA revealed significant increase in the number of TUNEL (+ve) cells in scramble SsiRNA transfected cultures, which was significantly reduced in NP1-siRNA transfected neurons. Data are expressed as percent TUNEL/DAPI cells (mean SEM, $n=8 ;{ }^{* *} p<0.01$ compared to control normoxia; $+p<0.01$ compared to OGD scramble siRNA group).

death when added to NP1-KO cultures (Figure 5C1), which otherwise showed neuroprotection against OGD. Our findings confirm the specific involvement of NP1 in neuronal death and neuronal survival in the absence of NP1 protein expression. Taken together, our results clearly demonstrate the role of extracellular NP1 in ischemic neuronal death.

Presence of NP1 protein in the CM, but not the NP2, is involved in OGD-induced neurotoxicity

To further validate the specificity of NP1 in neurotoxicity, we added CM from OGD-exposed NP2-KO cultures (NP2-KO OGD CM) to a subset of WT normal cortical cultures. As we have observed in Figure $4 \mathrm{~B}$ and $5 \mathrm{~A}$, the LDH release cytotoxicity assay showed significantly increased neurotoxicity $(\mathrm{p}<0.01)$ when the NP2-KO OGD
CM was added to normal WT cortical cultures (Figure 6 B), similar to that observed in case of WT-OGD CM (Figure 6A). Western blot analysis of NP2-KO OGD CM revealed increased levels of NP1 protein present in the CM from OGD-exposed NP2-KO cortical cultures. Our results clearly delineate the involvement of NP1, but not the NP2, in ischemic neuronal death following OGD, suggesting the specificity of NP1 in the neuronal injury mechanisms.

\section{Surface clustering of extracellular NP1 with AMPA}

\section{GluR1 receptors}

Previously we reported that OGD exposure promotes redistribution of AMPA GluR1 receptors at the postsynaptic membrane and significantly increased the NP1-GluR1 interactions at synaptic sites as evidenced by the higher 

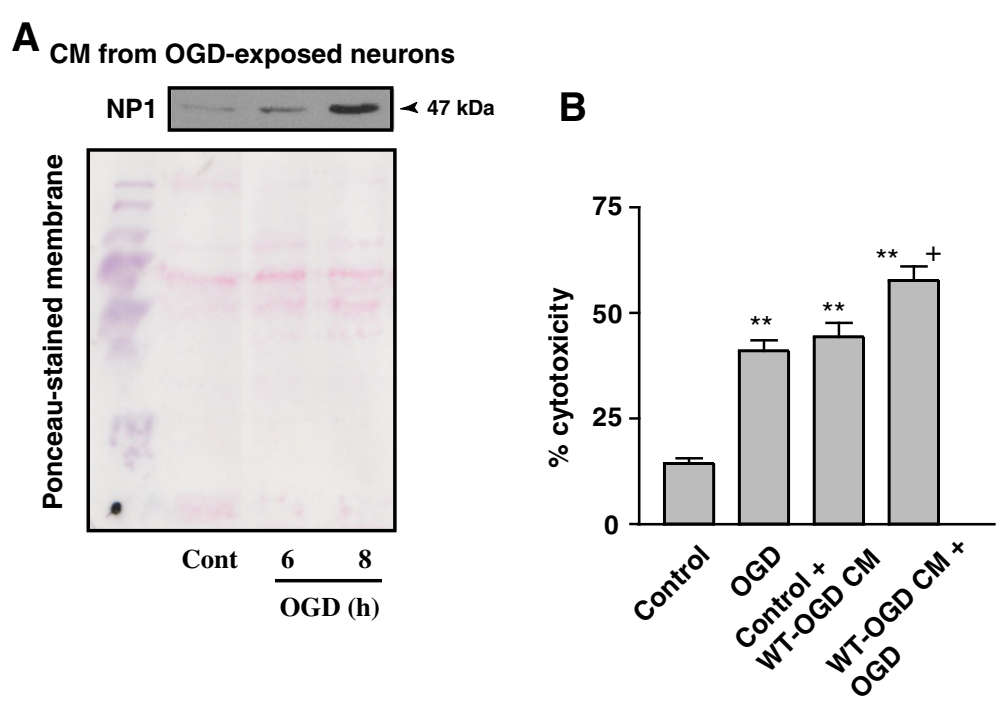

Figure 4 OGD-conditioned culture media caused increased neuronal death in cortical neuronal cultures. Primary cortical cultures at DIV 12 were exposed to OGD and conditioned media (CM) were collected, centrifuged and concentrated as described in the methods. A) Western blot analysis of CM from OGD-exposed WT primary cortical cultures showed OGD time-dependent increase of NP1 protein levels. B) This CM of OGD exposed WT cultures (WT-OGD CM) was added to a subset of control cortical cultures for $24 \mathrm{~h}$ (Control + WT-OGD CM). In addition, another subgroup of cells was also exposed to $6 \mathrm{~h}$ of OGD (WT-OGD CM + OGD). Data shown are mean SEM ( $\mathrm{n}=8 \mathrm{in}$ each group) and repeated two times, ${ }^{* *} p<0.01$ vs. normoxia controls; $+p<0.01$ vs. OGD only and Control + WT-OGD CM group.

percentage of NP1/PSD-95 co-localization and coclustering with GluR1 under similar OGD conditions [22]. We asked if extracellular release of NP1 following OGD interacts with GluR1. Live immunostaining with both NP1 and GluR1 antibodies showed intense increase of NP1GluR1 co-clustering in the dendrites and axons of the OGD-exposed cortical neurons compared to the normoxia controls (Figure 7). Our results suggest a relationship between extracellular release of NP1 following NP1 induction and synaptic clustering of GluR1, which in turn promote OGD-induced neuronal death.

\section{Discussion}

We found increased levels of NP1 protein in the CM from OGD-exposed WT primary cortical neuronal cultures, and this CM caused substantial neurotoxicity when added to control cortical cultures. Most strikingly, the CM from OGD-exposed NP1-KO cortical cultures did not induce neurotoxicity to normal cortical cultures under identical conditions, and that NP1-KO cells maintained characteristic features of healthy neuronal morphology with intact processes. First, we found induction of NP1 following OGD exposure of cortical neurons and neuronal death, whereas, inhibition of NP1 expression by NP1-specific siRNA exerts neuroprotection against OGD. Our findings suggest that NP1, being a secretory protein, after induction following OGD undergoes release from neurons and this extracellular NP1 is involved in ischemic neuronal death. Here, we present evidence that extracellular secretion of NP1 protein following exposure to OGD is necessary to induce ischemic neuronal death, suggesting a new extracellular prodeath function of NP1.

Previously we have reported a role for NP1 in neonatal hypoxic-ischemic brain injury and subcellular localization of NP1 in to mitochondria enhanced mitochondriamediated neuronal death [6-8]. We also reported interactions of NP1with excitatory AMPA GluR1 receptors and direct regulation of surface GluR1 expression and synaptic clustering of NP1 with GluR1 [22]. These findings suggest the possibility that presynaptic NPR which binds to NP1; allowing NP1 to trans-synaptically attach to the extracellular domain of GluR1 at the postsynaptic specialization, thereby facilitating glutamate binding and, thus, enhancing excitotoxicity. In contrast, NP1-KO neurons showed reduced cytotoxicity by limiting synaptic GluR1 cluster formation due to absence of extracellular NP1 at synaptic sites. We also found that this clustering activity involves physical association between NP1 and GluR1, and that NP1 exhibited profound synaptic co-clustering with GluR1 following OGD [22]. Thus, it is possible that extracellular NP1 may disrupt inter-neuronal synaptic activity, which possibly contributes to the neuronal death in hypoxicischemic brain injury via clustering with GluR1.

Based on cDNA sequence, NP1 is predicted to be secreted protein [9], raising the possibility that it is present on the neuronal surface that enhances the toxicity of neuronal cells under hypoxic-ischemic stress. We performed a series of experiments to evaluate the importance of extracellular NP1 in hypoxic-ischemic neuronal death. Hypoxic- 

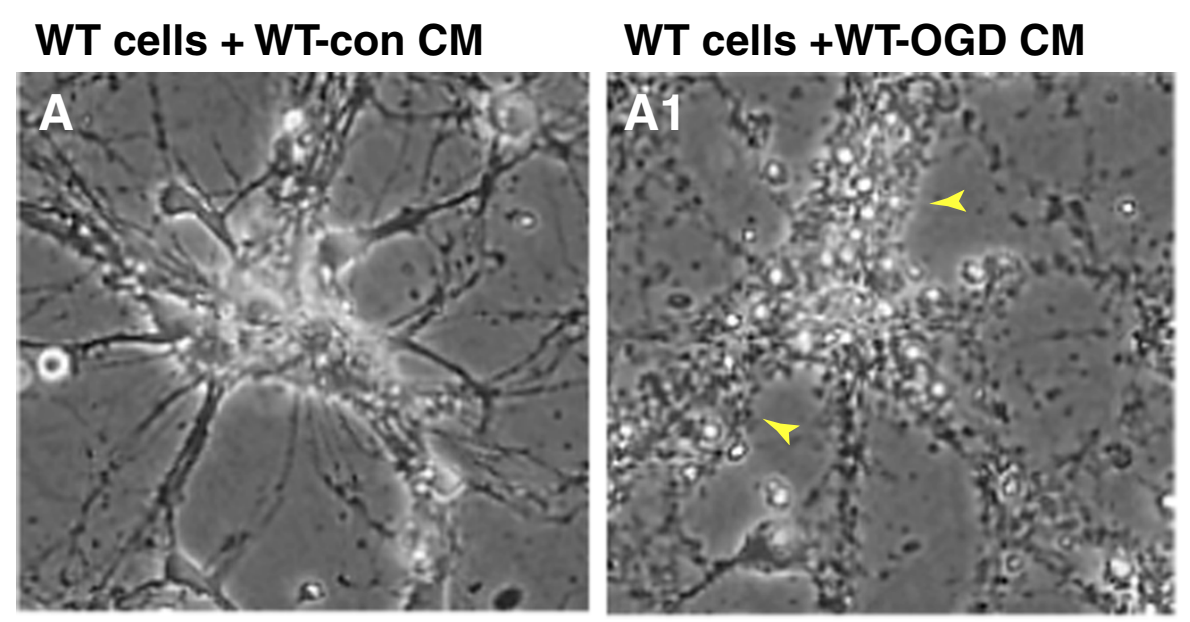

WT cells +NP1-con CM

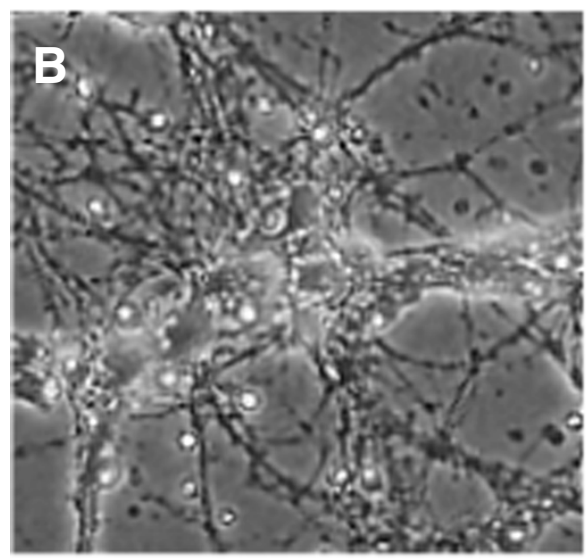

NP1-KO cells+WT-con CM

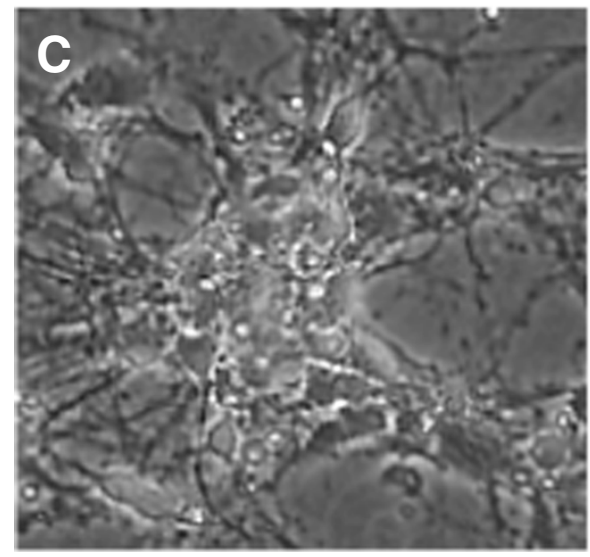

\section{WT cells+NP1-KO OGD CM}

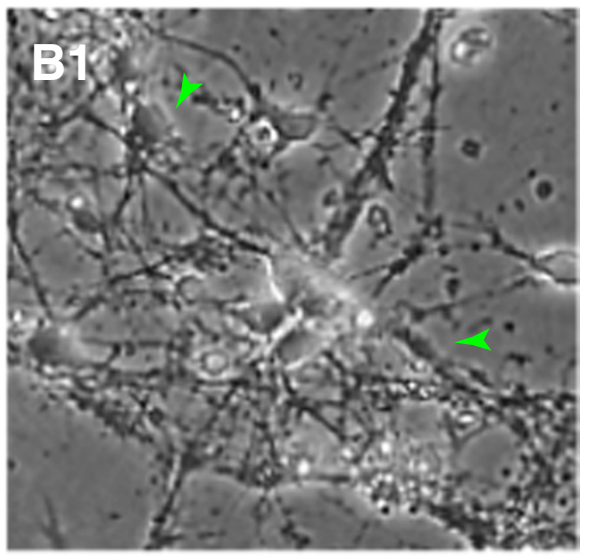

\section{NP1-KO cells+WT-OGD CM}

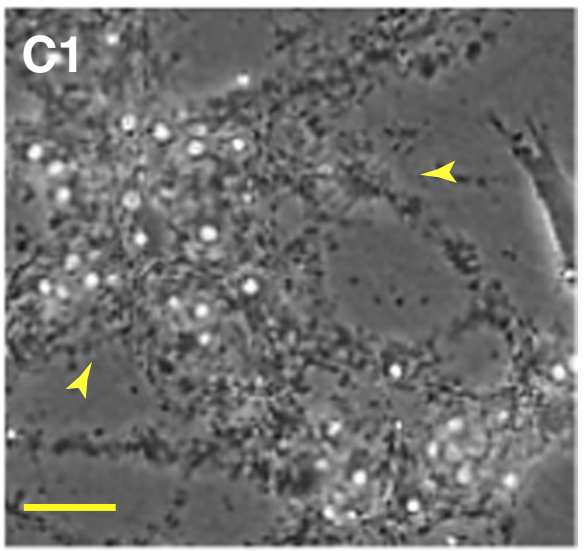

Figure 5 Absence of NP1 protein in NP1-KO OGD conditioned medium does not cause neuronal death in WT normoxia cortical cultures. Morphological evidence of degenerated cell bodies and processes reveal that WT OGD CM induced neuronal degeneration in control WT cultures (A-A1) whereas, OGD conditioned NP1-KO CM did not cause cell death in WT cultures (B-B1). In contrast, NP1-KO cortical cultures, which are protected against OGD, showed neuronal death when treated with WT-OGD CM (C-C1). Representative light microscopic images are shown, $n=6$, Scale, $100 \mu \mathrm{m}$. 
A

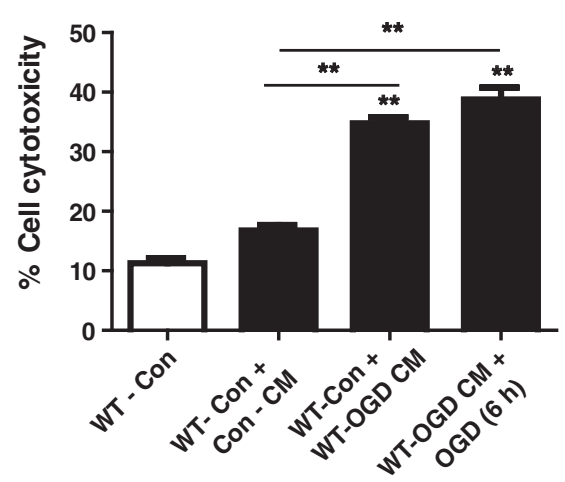

B

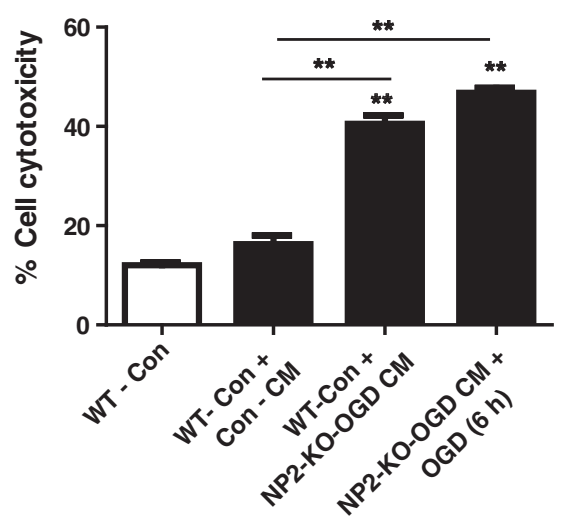

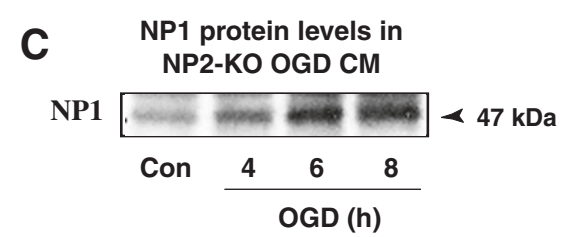

Figure 6 NP2-KO OGD conditioned medium caused neurotoxicity and death in WT normoxia cortical cultures. Quantification of cell death by LDH release assay revealed significantly higher percentage of cell death when WT-OGD CM was added to WT control cultures (A). B) The NP2-KO OGD CM caused similar extent of cytotoxicity when added to the WT control cultures as compared to control CM (Con-CM). Similarly, the NP2-KO OGD CM in combination with OGD further enhanced cell death. Data shown are mean SEM ( $\mathrm{n}=8$ in each group) and repeated two times, ${ }^{* *} \mathrm{p}<0.01$ vs. normoxia controls. C) Western blot analysis of OGD exposed NP2-KO CM showed OGD time-dependent increased levels of NP1 protein present in the NP2-KO OGD CM, suggesting NP1, but not the NP1, contributes to neuronal death. Representative bands are shown.

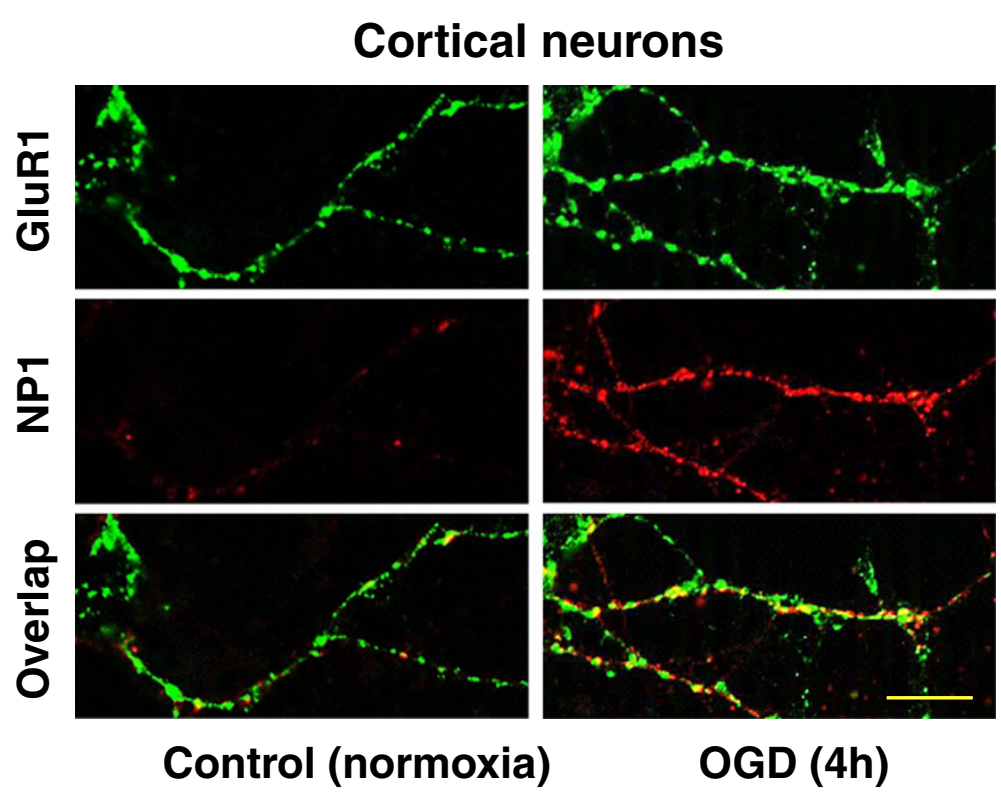

Figure 7 Extracellular release of NP1 protein following OGD enhances interaction between NP1 and GluR1 at the synaptic sites. Live immunostaining of DIV 12 primary cortical neurons with NP1 and GluR1 antibodies following OGD (4 h). Immunofluorescence microscopy and merged images show increased number of NP1 (red) and GluR1 (green) co-localized clusters (yellow) at cortical dendrites and axons. Representative images are shown, $n=6$, Scale, $100 \mu \mathrm{m}$. 
ischemic neuronal injury is triggered by the activation of glutamatergic excitotoxicity cascade [32] and several downstream cytotoxic pathways $[1,33]$. It appear from our present findings that NP1 is induced and released following OGD and recruited to surface GluR1 subunits to form clusters at excitatory synapses, and increased NP1-GluR1 interactions sensitize neurons to OGD- induced neuronal death. These members of the long-pentraxin family, NP1 and NP2, are exclusively expressed in the central neurons and are secretory from cells upon pathological stress. To further delineate the role of secretory NPs in neuronal death, we found that it is NP1 protein, not the NP2, is involved in neuronal death as evident by our findings that the CM from the OGD-exposed NP2-KO cortical cultures caused neuronal death when added to the normal cortical cultures similar to that induced by WT-OGD CM. The role of NP1 in neuronal death is also evidenced by the presence of increased amount of NP1 protein in the NP2$\mathrm{KO} \mathrm{CM}$ that caused neurotoxicity.

\section{Conclusions}

Collectively, our results argue that extracellular secretion of NP1 following OGD provides a mechanism that potentiates ischemic neuronal death possibly via AMPA GluR1mediated function at the excitatory synapses, and that NP1, not its family member NP2, is involved in neuronal death mechanisms. Our results suggest NP1 as a practical target for preventing ischemic neuronal death.

\begin{abstract}
Abbreviations
AMPA: a-amino-3-hydroxy-5-methyl-4-isoxazole-propionic acid; CM: Conditioned medium; EBSS: Earl s balanced salt solution; HI: Hypoxia-ischemia; OGD: Oxygen glucose deprivation; RT-qPCR: Real-time quantitative polymerase chain reaction; NP1: Neuronal pentraxin 1; NP2: Neuronal pentraxin 2; NMDA: N-methyl-D-aspartate.
\end{abstract}

\section{Competing interests}

The authors declare that they have no competing interests.

\section{Authors contributions}

ST made contributions to the primary neuronal cultures and established the OGD model, Western blot analysis, RT-qPCR, cytotoxicity assay. MAH conceived the study, participated in the study design and coordination, data and statistical analyses, figure formatting and writing the manuscript. Both authors read and approved the final manuscript.

\section{Acknowledgements}

This work was supported by the National Institutes of Health grant R01NS046030-09 (MAH) from the National Institute of Neurological Disorders and Stroke. The content is solely the responsibility of the authors and does not necessarily represent the official views of the National Institute of Neurological Disorders and Stroke or the National Institutes of Health.

\section{Author details}

${ }^{1}$ Hugo W. Moser Research Institute at Kennedy Krieger, Baltimore, MD 21205, USA. ${ }^{2}$ Department of Neurology, Johns Hopkins University School of Medicine, Baltimore, MD 21205, USA. ${ }^{3}$ Department of Neurology, The Kennedy Krieger Institute, 707 North Broadway, Room 400-N, MD 21205 Baltimore, USA.

Received: 27 August 2014 Accepted: 11 December 2014

Published online: 20 December 2014

\section{References}

1. Choi DW, Rothman SW: The role of glutamate neurotoxicity in hypoxicischemic neuronal death. Ann Rev Neurosci 1990, 13:171 182.

2. Barks JD, Silverstein FS: Excitatory amino acids contribute to the pathogenesis of perinatal hypoxic-ischemic brain injury. Brain Pathol 1992, 2(3):235 243

3. Portera-Cailliau C, Price DL, Martin L: Non-NMDA and NMDA receptor mediated excitotoxic neuronal deaths in adult brain are morphologically distinct: further evidence for an apoptosis-necrosis continuum. J Comp Neurol 1997, 378:88 104

4. Hollman M, Heinemann S: Cloned glutamate receptors. Ann Rev Neurosci 1994, 17:31 108

5. D Mello SR, Galli C, Ciotti T, Calissano P: Induction of apoptosis in cerebellar granule neurons by low potassium: inhibition of death by insulin-like growth factor I and CAMP. Proc Natl Acad Sci U S A 1993, 90(23):10989 10993.

6. Al Rahim M, Thatipamula S, Hossain MA: Critical role of neuronal pentraxin 1 in mitochondria-mediated hypoxic-ischemic neuronal injury. Neurobiol Dis 2013, 50:59 68.

7. Hossain MA, Russell JC, O Brien R, Laterra J: Neuronal pentraxin 1: a novel mediator of hypoxic-ischemic injury in neonatal brain. J Neurosci 2004, 24(17):4187 4196.

8. Russell JC, Kishimoto K, O Driscoll C, Hossain MA: Neuronal pentraxin 1 induction in hypoxic-ischemic neuronal death is regulated via a glycogen synthase kinase-3alpha/beta dependent mechanism. Cell Signal 2011, 23(4):673 682.

9. Schlimgen AK, Helms JA, Vogel H, Perin MS: Neuronal pentraxin, a secreted protein with homology to acute phase proteins of the immune system. Neuron 1995, 14(3):519 526.

10. Hsu YC, Perin MS: Human neuronal pentraxin II (NPTX2): conservation, genomic structure, and chromosomal localization. Genomics 1995, 28(2):220 227

11. Dodds DC, Omeis IA, Cushman SJ, Helms JA, Perin MS: Neuronal pentraxin receptor, a novel putative integral membrane pentraxin that interacts with neuronal pentraxin 1 and 2 and taipoxin-associated calcium-binding protein 49. J Biol Chem 1997, 272(34):21488 21494.

12. Tsui CC, Copeland NG, Gilbert DJ, Jenkins NA, Barnes C, Worley PF: Narp, a novel member of the pentraxin family, promotes neurite outgrowth and is dynamically regulated by neuronal activity. J Neurosci 1996, 16(8):2463 2478.

13. Goodman AR, Cardozo T, Abagyan R, Altmeyer A, Wisniewski HG, Vilcek J: Long pentraxins: an emerging group of proteins with diverse functions. Cytokine Growth Factor Rev 1996, 7(2):191 202.

14. Omeis IA, Hsu YC, Perin MS: Mouse and human neuronal pentraxin 1 (NPTX1): conservation, genomic structure, and chromosomal localization. Genomics 1996, 36(3):543 545.

15. Gewurz $H$, Zhang $X H$, Lint TF: Structure and function of the pentraxins. Curr Opin Immunol 1995, 7(1):54 64.

16. Bottazzi B, Vouret-Craviari V, Bastone A, De Gioia L, Matteucci C, Peri G, Spreafico F, Pausa M, DEttorre C, Gianazza E, Tagliabue A, Salmona M, Tedesco $F$, Introna M, Mantovani A: Multimer formation and ligand recognition by the long pentraxin PTX3. Similarities and differences with the short pentraxins C-reactive protein and serum amyloid P component. J Biol Chem 1997, 272(52):32817 32823.

17. Kirkpatrick LL, Matzuk MM, Dodds DC, Perin MS: Biochemical interactions of the neuronal pentraxins. Neuronal pentraxin (NP) receptor binds to taipoxin and taipoxin-associated calcium-binding protein 49 via NP1 and NP2. J Biol Chem 2000, 275(23):17786 17792.

18. O Brien RJ, Xu D, Petralia RS, Steward O, Huganir RL, Worley P: Synaptic clustering of AMPA receptors by the extracellular immediate-early gene product Narp. Neuron 1999, 23(2):309 323.

19. OBrien $R, X u$ D, Mi R, Tang X, Hopf C, Worley P: Synaptically targeted narp plays an essential role in the aggregation of AMPA receptors at excitatory synapses in cultured spinal neurons. J Neurosci 2002, 22(11):4487 4498.

20. Hossain MA: Molecular mediators of hypoxic-ischemic injury and implications for epilepsy in the developing brain. Epilepsy Behav 2005, 7(2):204 213.

21. Hossain MA: Hypoxic-ischemic injury in neonatal brain: involvement of a novel neuronal molecule in neuronal cell death and potential target for neuroprotection. Int J Dev Neurosci 2008, 26(1):93 101.

22. Al Rahim M, Hossain MA: Genetic deletion of NP1 prevents hypoxicischemic neuronal death via reducing AMPA receptor synaptic localization in hippocampal neurons. J Am Heart Assoc 2013, 2(1):e006098. 
23. Watson A, Eilers A, Lallemand D, Kyriakis J, Rubin LL, Ham J:

Phosphorylation of C-Jun is necessary for apoptosis induced by survival signal withdrawal in cerebellar granule neurons. J Neurosci 1998, 18(2):751 762.

24. Sharma J, Johnston MV, Hossain MA: Sex differences in mitochondrial biogenesis determine neuronal death and survival in response to oxygen glucose deprivation and reoxygenation. BMC Neurosci 2014, 15:9.

25. Hossain MA, Bailone JC, Gomez R, Laterra J: Neuroprotection by scatter factor/hepatocyte growth factor and FGF-1 in cerebellar granule neurons is phosphatidylinositol 3-kinase/Akt-dependent and MAPK/CREB-independent. J Neurochem 2002, 81(2):365 378.

26. Sharma J, Nelluru G, Wilson MA, Johnston MV, Hossain MA: Sex-specific activation of cell death signaling pathways in cerebellar granule neurons exposed to oxygen glucose deprivation followed by reoxygenation. ASN Neuro 2011, 3(2):85 97.

27. Nonaka S, Hough CJ, Chuang DM: Chronic lithium treatment robustly protects neurons in the central nervous system against excitotoxicity by inhibiting N-methyl-D- aspartate receptor-mediated calcium influx. Proc Natl Acad Sci U S A 1998, 95(5):2642 2647

28. Russell JC, Blue ME, Johnston MV, Naidu S, Hossain MA: Enhanced cell death in MeCP2 null cerebellar granule neurons exposed to excitotoxicity and hypoxia. Neuroscience 2007, 150(3):563 574.

29. Hossain MA, Russell JC, Miknyoczki S, Ruggeri B, Lal B, Laterra J: Vascular endothelial growth factor mediates vasogenic edema in acute lead encephalopathy. Ann Neurol 2004, 55(5):660 667.

30. Russell JC, Szuflita N, Khatri R, Laterra J, Hossain MA: Transgenic expression of human FGF-1 protects against hypoxic-ischemic injury in perinatal brain by intervening at caspase-XIAP signaling cascades. Neurobiol Dis 2006, 22(3):677 690

31. Semenza GL: Hypoxia-inducible factor 1: oxygen homeostasis and disease pathophysiology. Trends Mol Med 2001, 7(8):345 350.

32. Portera-Cailliau C, Price DL, Martin LJ: Excitotoxic neuronal death in the immature brain is an apoptosis-necrosis morphological continuum. J Comp Neurol 1997, 378(1):70 87.

33. Johnston MV, Trescher WH, Ishida A, Nakajima W: Neurobiology of hypoxic-ischemic injury in the developing brain. Pediatr Res 2001, 49(6):735 741

\section{Submit your next manuscript to BioMed Central and take full advantage of:}

$\otimes$ Convenient online submission

$\otimes$ Thorough peer review

$\nabla$ No space constraints or color $\nabla$ gure charges

$\otimes I m m e d i a t e$ publication on acceptance

Q Inclusion in PubMed, CAS, Scopus and Google Scholar

$\otimes$ Research which is freely available for redistribution 